\title{
Dynamic Behavior of Tyre Tread Block
}

\author{
${ }^{1}$ K. Sridharan and ${ }^{2}$ R. Sivaramakrishnan \\ ${ }^{1}$ Department of Rubber and Plastics Technology, \\ ${ }^{2}$ Department of Production Technology \\ Anna University (MIT Campus), Chennai- 600 044, India
}

Received 2012-06-04, Revised 2012-07-16; Accepted 2012-07-27

\begin{abstract}
The Tyre tread pattern is the arrangement of blocks, grooves and voids, sipes and channels designed into the tread to enhance its grip on the road. Tread is the uppermost part of any tyre which contact into the road and it has its own performance towards mileage, traction, low noise and heat built up properties. It would be meaningful to conduct an extended analysis on the tyre tread blocks for its performance in static and dynamic condition to predict its behavior and wear of tread block in on-road condition. The Finite Element software Abaqus is used for the present analysis of the tread block and its behavior was studied on two different contact surfaces. The tread block is modeled in six different shapes and analyzed for is performance. The deformation stress strain characteristic of different blocks is studied which will be useful in deciding the contact behavior, friction and road grip. The regular shape/geometry has common behavior and the mixed type geometry shows a distinguished variation in the analysis. The dynamic stiffness and deformed shape was analyzed in this study and it has its own effect in tyre design. The tread block dynamics study by geometric shape would be vital in tread pattern optimization to enhance the traction, better hydroplaning and rolling resistance under all operating conditions.
\end{abstract}

Keywords: Tread Pattern Optimization, Static and Dynamic, Block Dynamics, Geometric Shape

\section{INTRODUCTION}

Friction is present in all mechanical systems and originates at an interface between bodies, which are in contact with each other. This contact enables solids to transmit forces through the surfaces. The resultant force can be resolved into a normal and a tangential force. Forces can only be transmitted if there is contact and therefore the contact area plays an import role in describing friction aspects.

\subsection{Literature Review on Friction and Wear:}

The friction force between rubber and a rough surface has two contributions commonly described as the adhesion and hysteretic components (Moore, 1972). Several theories have been developed to describe surface roughness and the relation to the real contact area. Grosch (1963) indicated that friction is due to energy dissipated when rubber is compressed and released by asperities. Schallamach (1971) 2D model is a generalized theory that predicts the buckling effects, known as Schallamach waves which occurs at very smooth surfaces. There are four different sources of non-linearity in solid mechanics which includes geometrical, material, force boundary conditions and displacement boundary conditions. In the tyre tread block contact phenomenon the above stated non-linearity are highly concerned as they are not constant and its variation is highly dependent on pavement.

The present work concentrates on the contact phenomena and to the dynamic behavior of the external tyre elements (i.e. tread and blocks), which are those directly at the interface with the ground. According to $\mathrm{M}$. Brusarosco the Knowledge of contact forces during sliding will allow us to understand how much influence on breaking performance is due to tread block dynamic properties (Brusarosco et al., 2011).

There have been substantial studies on the tyre tread pattern design for the purpose of technical performance and energy savings (Majumdar, 2011). Samir Majumdar in his review paper stated tyre tread pattern are the arrangement of blocks, grooves, sipes and channels designed into the tread to enhance its grip, the part which contacts the road. The tread in the contact patch undergoes a significant stress and in general all tread compounds demand on following parameters: 
- Wear resistance

- Low heat built up

- Low fuel consumption

- Resistance to chip and cut

- Good road grip (traction)

There are four important stages in moving through the footprint as discussed by the (Alan et al., 1999).

Stage 1: The element approaches the pavement.

Stage 2: The element makes contact and becomes "frictionally adhered" to the pavement. As the tyre continues to roll forward, the location of the element with respect to the center of tyre rotation changes, this distance first decreases and then increases as the element moves backward. A combination of lateral or longitudinal (or combined) tangential force (FT) arises at the interface due to traction. At the same frictional adhesion on the pavement of the element cannot continue and slip or sliding motion occurs.

The frictional work or energy $\mathrm{FE}$ is generated and given by Eq. 1:

$\mathrm{FE}=\int \mathrm{FT} * \mathrm{dl}$.

Where:

$$
\text { dl-slip or sliding distance. }
$$

Stage 3: Completion of sliding occurs.

Stage 4: The element leaves the pavement.

Thus the above Fig. 1 clearly illustrates the phenomenon of tread element contact over the pavement. It has been inferred that the factors for tread wear also has importance on tread pattern (groove void) feature and geometric shape or aspect ratio in the view of tyre construction. Now with the development of all seasonal tyres by the tyre manufactures with improved tread pattern design and its feature it could be interesting to study the dynamic behavior of a tread block. The all season tyre includes a high level of care in designing tread patterns because tyre without tread is quite dangerous on wet roads. In dry roads the tyre without tread would be an effective one.

The relationship between wear and frictional energy is given as Eq. 2:

$\mathrm{RW}=\mathrm{A} * \mathrm{FE}$

where, RW-Rate of wear i.e amount of rubber lost from a unit tread surface per tyre revolution, A-Abradability, FEFrictional work or Energy per unit area. per revolution.

The Fig. 1 clearly illustrates the phenomenon of contact mechanism of the tread block and the equations exhibit the contact of tyre over the pavement and its wear rate for its Finite Element studies for setting the loads and boundary conditions.

\subsection{Tyre Tread Block Mechanics}

This study focuses on the interaction between a Tread block and the road surface. A three-dimensional model of this contact problem is required. The concept of a rolling tyre in contact with the road surface is a complicated problem with many non-linearity such as large deformations, non-linear boundary constraints, non-linear tyre and pavement material characteristics and complex geometry and structure of the tyre and the road.

When the contact between a tyre and a road is observed at increasing magnification, the area of real contact will monotonically decrease. At some high magnification, typically corresponding to a resolution (or wavelength $\lambda c$ ) of order micrometer, the stresses and the temperature increase during slip will be so high that bonds in the rubber will rupture resulting in, e.g. Micrometer sized cracks and wear processes. When the tyre temperature increases the wear rate usually increases strongly. This is due to the reduction in the internal damping in the rubber and consequently strong increase in the crack tip propagation velocity.

The tread pattern is a source of anisotropy in tyre tread layer. Tread blocks may be compressed and bent so much that the vertical load is transmitted just by frontal edges of the tread pattern blocks. Lateral displacements of the surface of tread layer become substantially greater than those in radial directions due to considerable bending and stretching tread blocks.

Higher modulus and smaller groove depth reduce tangential mobility of tread blocks. Tread pattern provides flexibility to tread rubber blocks and plays a very important role in tyre response to tangential loading. Rubber modulus under the same conditions depends on rubber blend composition. It changes during the tyre life and its instant values depend on temperature. Groove depths decrease due to tread wear and at the same time the stiffness of the tread rubber decreases due to the temperature increase. As a rule, however, $\mathrm{E}$ is increased in tread rubber aging.

In the tread/road interface local strains are influenced by the macroscopic and microscopic bumps and asperities on the road surface. Corresponding stresses may exceed the critical level of material strength. Then microscopic particles are torn out of the surface. This destructive process is manifested as the tread wear. Wear rate depends on tread rubber compound, road surface quality, interface temperature.

The present work focuses on the modeling of the tread block with a different geometry. The contact between rigid pavement and a rubber tread block with same contact area and different shapes i.e., same volumetric properties with different shape on a rigid surface is conducted using ABAQUS software. 

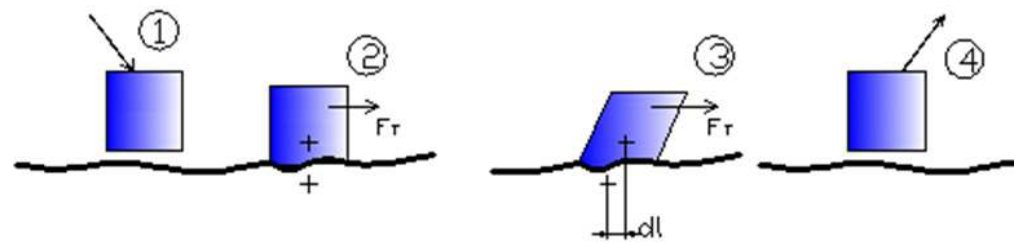

Fig. 1. Tread element approach

\subsection{Material Model}

Selecting the material model is one of the most extended and complex work in tyre modeling. The tread parts are commonly modeled using solid elements with a specific isotropic hyperelastic material model (Mooney Rivlin). Many theoretical models have been developed to characterize the mechanical behavior of rubber. One of the most important is the Mooney-Rivlin model as in Eq. 3:

$\mathrm{W}=\mathrm{C} 10(\mathrm{I} 1-3)+\mathrm{C} 01(\mathrm{I} 2-3)$

where, $\mathrm{C} 10$ and $\mathrm{C} 01$ are material constants and I1 and I2 are strain invariants. This model is extensively used for stress analysis of rubber components and is incorporated in most commercial FEA programs and is therefore used in the present study.

\section{MATERIALS AND METHODS}

The basic idea to conduct the research is to develop the tread block in various shapes and its geometrical property is analyzed in static and dynamic condition. The deformation behavior of the block is studied which will be useful to optimize the design.

\subsection{Experimental Tests for Material Property}

The tread is made from Natural rubber and/or blend of synthetic rubber (SBR/BR) mixed with carbon black/silica, oil, curative ingredients and different other chemicals and fillers. The rubber compound used in this study was NR and BR blend which is of a carbon blackfilled natural rubber compound typically employed in the production of Tyre Treads. The above recipe with standard formulation is mixed in two roll mill and cured in the hydraulic press.

\subsection{Determination of Material Constants}

The NR and BR blend tyre tread compound was prepared based on standard formulation. The tensile slab was molded in an electrically heated laboratory hydraulic press at $150^{\circ} \mathrm{C}$ for $15 \mathrm{~min}$. The mechanical properties Modulus, Tensile strength and elongation at break are evaluated.
To derive the material constants from the test data, the tension tests were carried out using a universal testing machine (AGS-2000G, Shimadzu). A uni-axial load was applied to the specimen in the cross-head at separation rate of $5 \mathrm{~mm} / \mathrm{min}$. (Natchimuthu, 2010). To predict the rubber behavior based on the Mooney-Rivlin model, the values of $\mathrm{C} 01$ and $\mathrm{C} 10$ must be determined. The graph for stretch ratio $1 / \lambda$ and $s / 2 *(\lambda-\lambda-2)$ have been plotted and the Mooney rivlin material constants are determined as shown in the below Fig. 2.

For the NR BR blended tread compound the three tensile specimens has been prepared according to ASTM standard and tested and the average values are taken for the analysis. The graph between stress and elongation was plotted as shown above in the Fig. 3 for the three specimens.

\subsection{Modeling of Tread Block}

The different models of a tread block which allows an exact depiction of its behavior under static and sliding/rolling conditions are modeled for displaying the dynamics of a tread block in a detailed way. First of all it is important to define the shape of the block with its width $\mathrm{w}$ and length 1 . In case of developing a 3D model, the third dimension $d$ is needed as well, furthermore the physical properties like mass, stiffness are required for predicting the exact block dynamics. The mould of the below shapes has been made in wire cut EDM process for its better geometrical accuracy. The patterns shown below are having same area across the depth with same mass and volume. The below Fig. 4 shows the different models of the tread blocks for Finite Element analysis and also for experimental work to find deformation and induced stress strains of the blocks using the image processing techniques in the further studies.

The normal force deforms the beam in longitudinal direction only and the lateral force operates on the transversal deflection. Due to the normal load, this material is deflected vertically as it goes through the contact patch and then springs back to its original shape after it leaves the contact patch. When the tyres are static (not rotating) the distribution of the normal load $F$, in the contact patch is symmetric with respect to the center of the contact patch. However, when the tyres are rotating, 
Sridharan, K. and R. Sivaramakrishnan / American Journal of Engineering and Applied Sciences 5 (2) (2012) 119-127

the normal load distribution is non-symmetric. Thus the study of tread block behavior under the static and dynamic condition would be effective in the pattern design and analysis.

\subsection{Finite Element Model}

The Finite element method is widely used in solving many engineering problems (Alshoaibi et al., 2009).

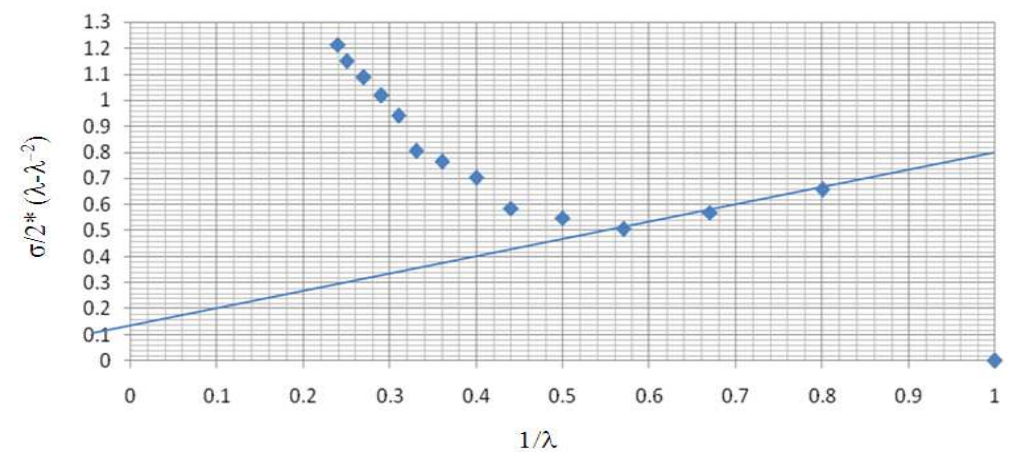

Fig. 2. Mooney rivlin plot

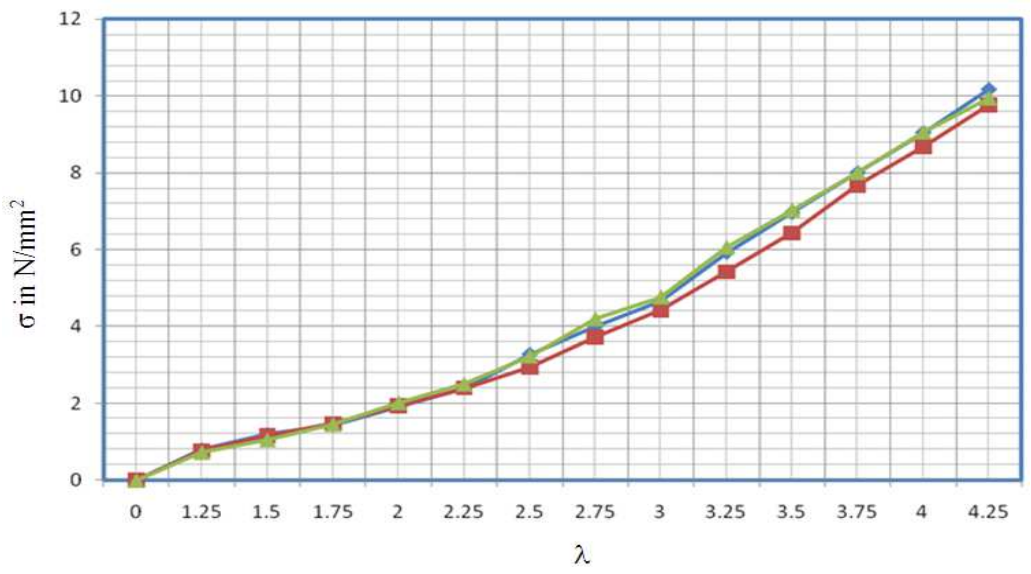

Fig. 3. Stress- stretch curve for samples

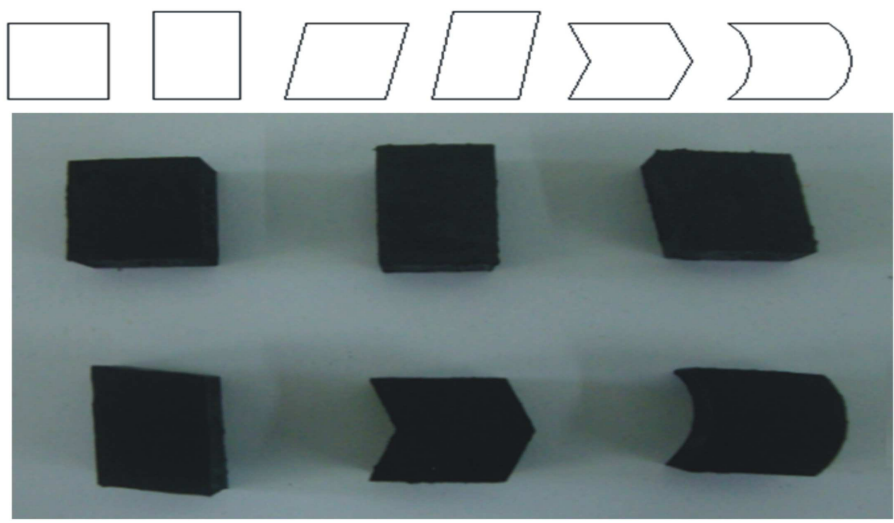

Fig. 4. Different tread blocks 


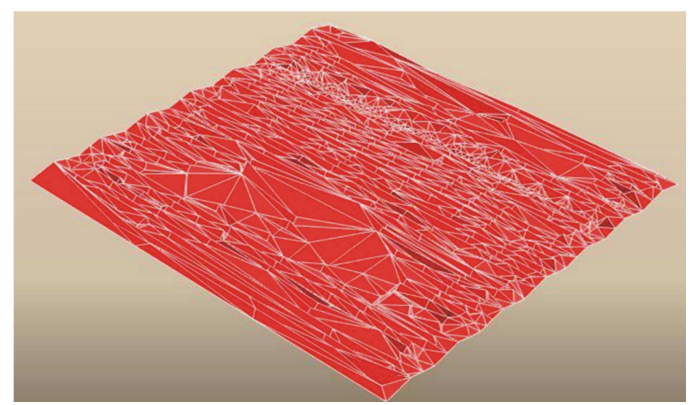

Fig. 5. Rigid road surface

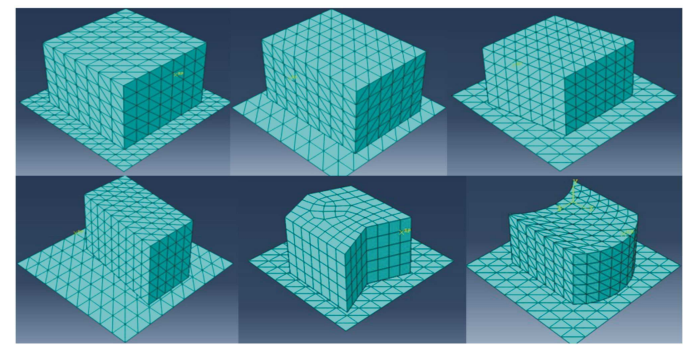

Fig. 6. Meshed tread blocks

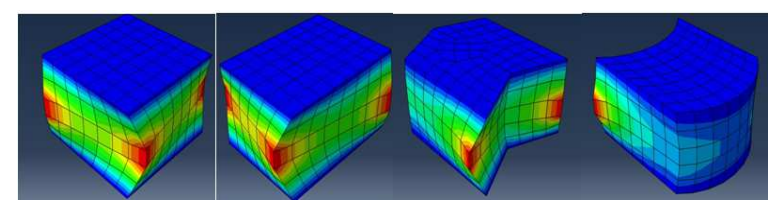

Fig. 7. Mode shapes of tread blocks

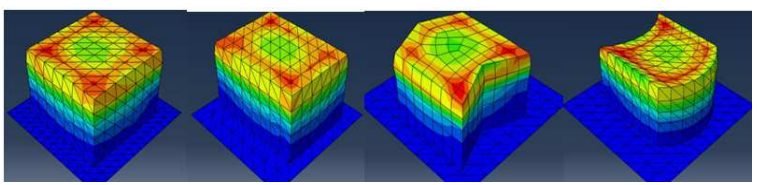

Fig. 8. Deformed shapes of tread blocks static load
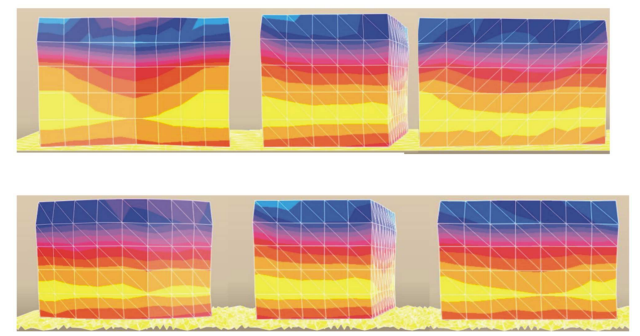

Fig. 9. Deformed shapes of tread blocks in pavements 1 and 2

The Contact between tyre and the road at static and dynamic situation was modeled and analyzed using ABAQUS Software. The dimensions of the tread block are chosen as such the area remains constant for square
Rectangular and all other mixed pattern block with a of depth $10 \mathrm{~mm}$. The Tetrahedral solid element with global size of 2 units is used for meshing the homogeneous solid rubber blocks. The two different pavement surfaces were created in ABAQUS, one perfect flat surface without any roughness and another with an average surface roughness of 500 microns as shown in the Fig. 5 below. The meshed model of the tread blocks are of different shapes are shown in the Fig. 6.

\subsection{Material Properties and Boundary Conditions}

The Mooney-Rivlin material model has been considered for this study and the values of $\mathrm{C} 10=0.387$ $\mathrm{MPa}, \mathrm{C} 01=0.232 \mathrm{MPa}$ which obtained by the experimental work from the tread formulation are used in for Analysis. Compressibility $\mathrm{d}=0.02274$, density $\mathrm{r}=1.091 \times 10-6 \mathrm{~kg} / \mathrm{mm} 3$.

The Rigid body (road) contact surface has been created in using discrete rigid type and assigned with a friction coefficient $\mu=0.5$. The static deformation of the tread block under the normal load was assigned with boundary conditions as the DOF along $\mathrm{Y}$ as zero and a uniform pressure load of $4.96 \mathrm{~N} \mathrm{~mm}^{-2}$ was applied on the top surface of the tread block. The combined Normal and tangential load for the tread block was assigned with the boundary condition as a tangential force of $0.496 \mathrm{~N} \mathrm{~mm}^{-2}$ along the area of contact with rigid road surface along with the normal pressure load. The analysis is carried out by normal static loading and combining the tangential force on different tread block and its performance was evaluated.

\subsection{Tread Block Dynamics}

Tyre tread blocks are permanently subjected to different driving states such as acceleration or braking as well as cornering (Nakajima, 2007). Even for a constant vehicle velocity the tread blocks experience an excitation resulting from the tyre deformation in the contact patch and from friction induced vibrations. The mode shapes of the tread block for the different patterns are obtained in Finite Element Analysis are shown in Fig. 7. The deformation of the block is shown in the Fig. 8 with its deformed shape under the static load condition over the contacting rigid body surface.

The contact time of a tread block for a vehicle velocity of $100 \mathrm{~km} \mathrm{~h}^{-1}$ takes about $5 \mathrm{~ms}$ during this contact the tread block passes a sticking and a sliding phase and finally snaps out (Moldenhauer and Kroger, 2010). This leads to an unsteady dynamic behavior of the tread blocks which has to be covered in models to achieve a realistic simulation. On the one hand, present tread block models usually show a low complexity in three-dimensional finite element approach with nonlinear material description. 
The friction coefficient is independent of contact pressure, size of contact area and also temperature, sliding speed. The adhesive and abrasive wear of the rubber particles over the contact surface causes the volumetric loss material under the dynamic condition. The stick-slip behavior of the tread block surface over the different pavement with different friction coefficient makes the process complicated in determining the wear rate.

The most important aspect of tyre-road interactions seems to be traction at the interface of their mutual rolling contact. Traction is essentially a friction process where tangential forces are transmitted and controlled by varying the relative motion of rolling and sliding between the contacting bodies. The relation between the actual contact area and apparent contact area of the tread blocks would be very effective in the traction of tyres other than ribbed pattern. The Fig. 9 illustrates the deformation plot of the tread blocks under two different pavements under the combined loads which are simulated for actual deformation studies of the tread blocks in on road conditions.

\section{RESULTS}

The tread block were simulated by combined normal and tangential load and was observed for its deformation behavior. The work is to understand the dynamics of tyre tread block and its contact behavior, which in turn linked to actual on-road condition. The frictional force on sliding against each other is largely independent of external influence. It is also proportional to normal reaction force between the two surfaces. By applying a constant Normal pressure load the static deformations was studied.

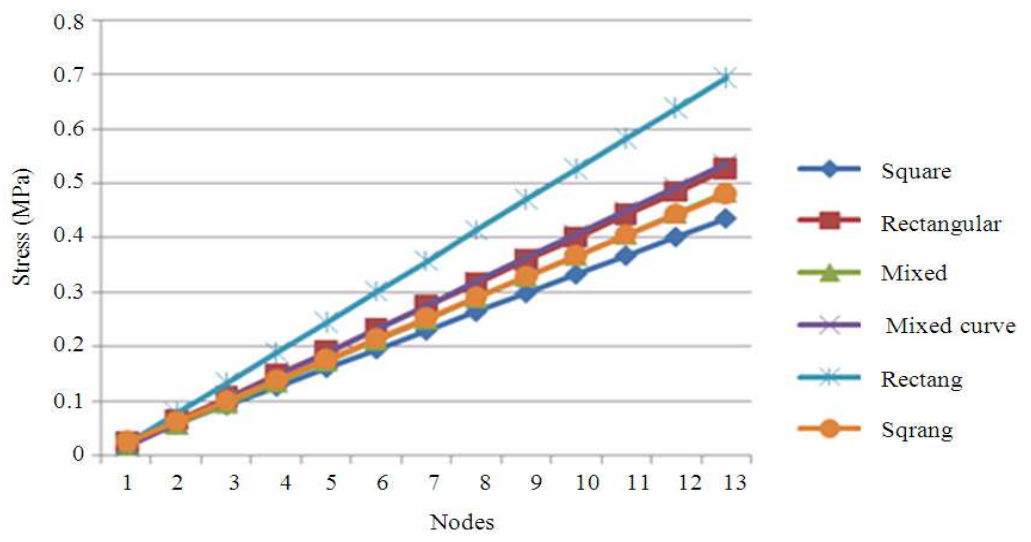

Fig. 10. Stress plot of the tread blocks under Normal loads pavement-1

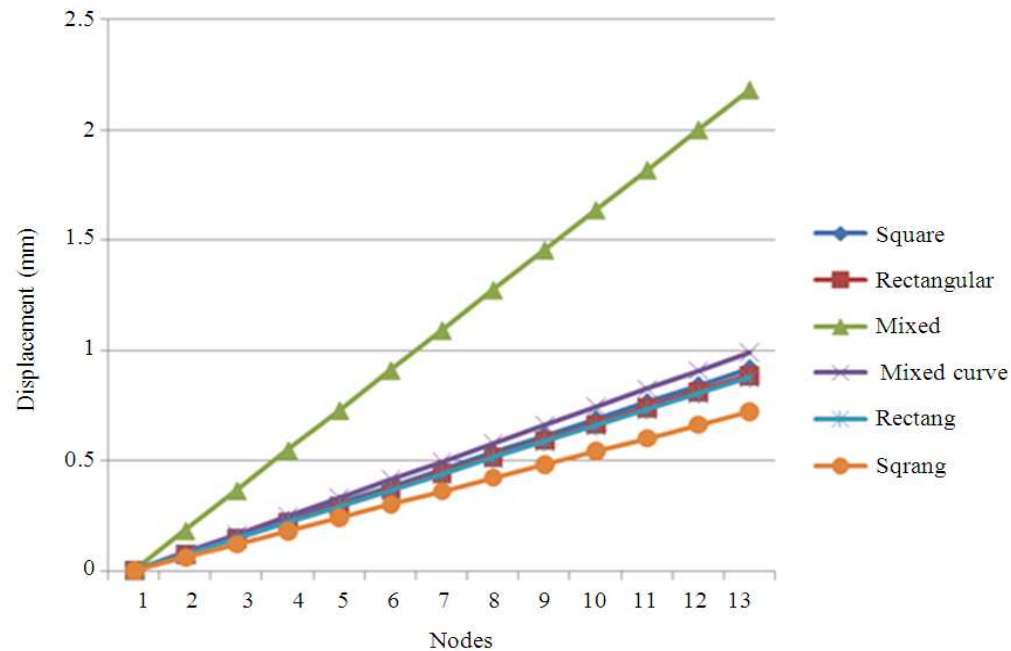

Fig. 11. Deformation plot under combined loads 
Sridharan, K. and R. Sivaramakrishnan / American Journal of Engineering and Applied Sciences 5 (2) (2012) 119-127

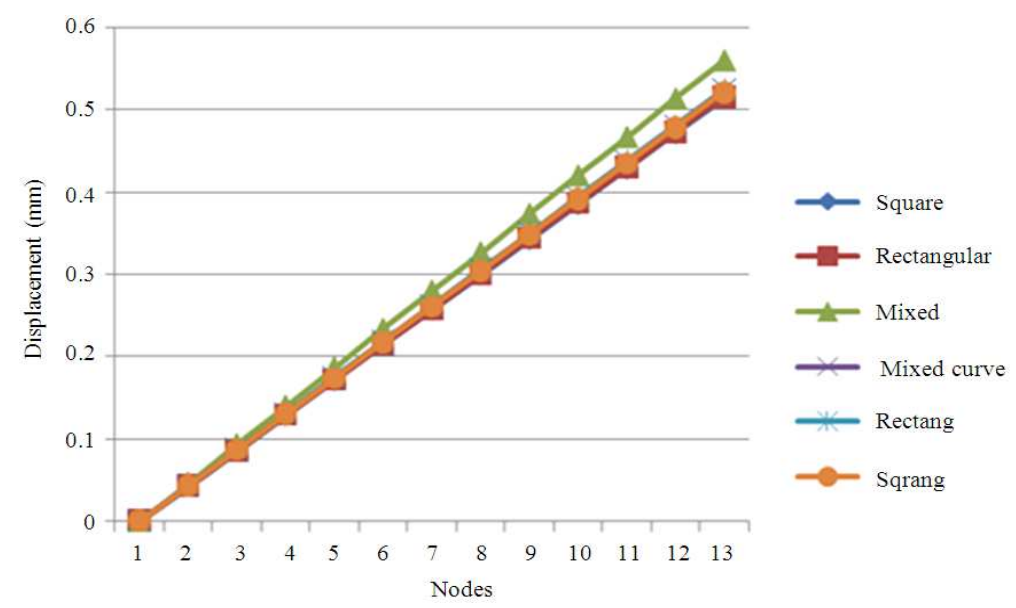

Fig. 12. Deformation plot combined loading pavement-1

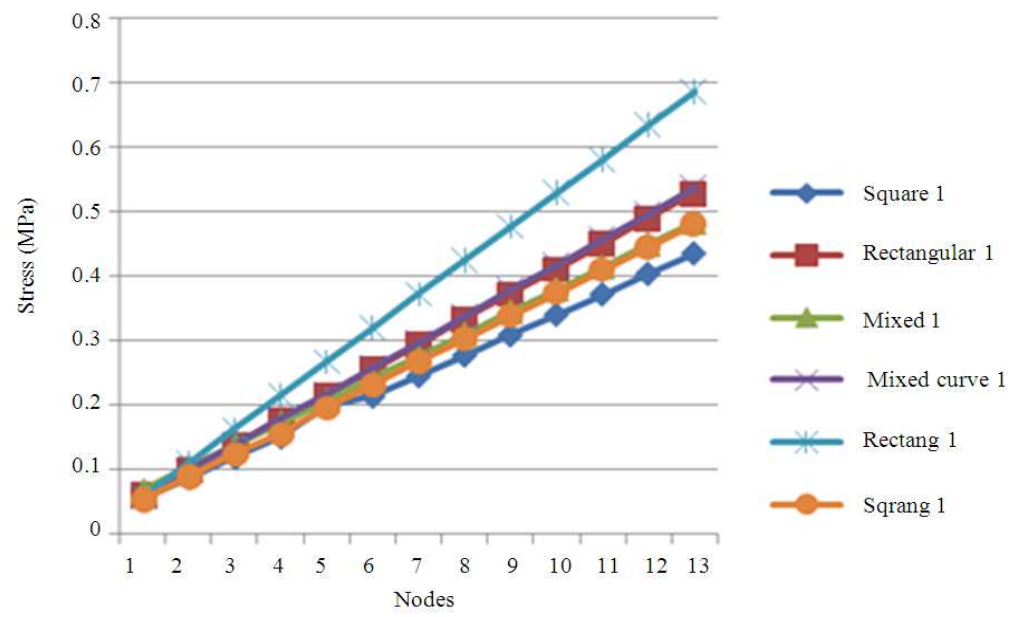

Fig. 13. Stress plot of the tread blocks under combined load pavement-1

For many technical applications rolling contacts of deformable bodies has to be found. For rapid changes of normal force and the lateral force the contact conditions cannot be described by steady models with constant contact area by representing the hyper elastic material behavior of the contacting body. The block is designed with loading area constant for all the tread blocks for the applied normal load and the stress values are mostly same in all patterns expect the rectangular pattern shown in Fig. 10 as its length is comparatively larger than other patterns which is inferred from the below graph. The square block pattern has mostly common behavior in deformation, stresses and strains for the applied Normal and lateral load. The regular rectangular pattern shows moderately high values of induced stress under the given load and boundary condition. But the mixed pattern model shows a distinguish value for the above mentioned parameters such as deformation stress and strain shown in Fig. 11. This may be because of the change in cross section from a regular geometry to mixed type geometry. The remaining geometries show a closure behavior as shown in the graph below.

The distinguished geometry in the tyre pattern is different in the actual contact area over the apparent contact area. The contact behavior on operating forces consistently varies as the contact surface geometry differs on road condition.

Under the static as well as dynamic condition due to normal loading and lateral loading the actual contact geometry differs from apparent contact geometry for the mixed pattern. For the remaining patterns tends to be very closure as shown in the graph above in Fig. 12. The mixed pattern shows distinguished values in deformation as well as stress induced as shown in Fig. 13 and 14 on two different pavements. 


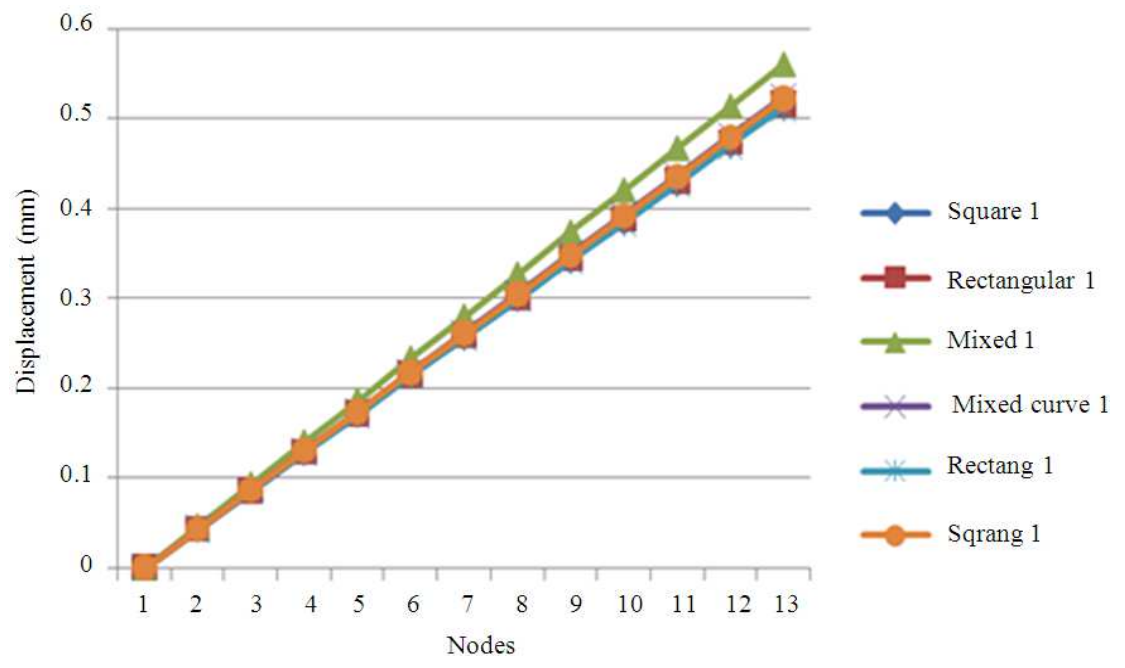

Fig. 14. Deformation plot of the tread blocks under combined load pavement-2

\section{DISCUSSION}

In both the loading cases normal as well as combined normal and tangential forces, the rectangular pattern shows a high induced stress values than the other pattern for the variable pavements. The mixed pattern shows distinguished values in deformation as well as stress induced on two different pavements. The friction between a tyre and a road surface is mainly due to the time dependent deformation of the rubber by the road surface. The interaction between tyre and the ground is implemented through the tread pattern. Different tread pattern will cause different pressure distribution and slipping distance on tyre. A finite element Analysis was carried with complex tread pattern which was modeled and analyzed to obtain more accurate tread block simulation under two different contact bodies.

\section{CONCLUSION}

It has been inferred that for the same apparent contact area of the tread pattern which is differed from the actual contact area on to the contact surface. The deformation of the tread blocks of six different geometries with unique mass properties have been studied and found out there is a distinguished variation in the displacement and induced stresses. Thus it proves the regular square and rectangular pattern on its dimension change has its own unique characteristics and shows almost same deformation and stress strain behavior. For the pattern changes other than square and rectangular shape has to be designed carefully as its behavior differs.
Thus the geometry change on the tread block has to be taken care for the mixed/irregular patterns as its behavior is distinct from the regular pattern.

\subsection{Future Work}

An Experimental set up using image processing has been under fabrication for the verification of the deformation of the tread block for its validation under the normal and shear load. The wear pattern of different tread block has to be estimated under general operating condition and the wear has to be estimated for all the tread patterns.

\section{REFERENCES}

Alan, G., T. Veith and G. Voild, 1999. Developed Tread width (Aspect Ratio): Their joint influence on wet traction. Rubber Chem. Technol., 72: 684-700.

Alshoaibi, A.M., A.K. Ariffin and M.N. ALmaghribi, 2009. Development of efficient finite element software of crack propagation simulation using adaptive mesh strategy. Am. J. Applied Sci., 6: 661666. DOI: 10.3844/ajassp.2009.661.666

Brusarosco, M., A. Cigada and S. Manzoni, 2011. Measurement and analysis of tyre and tread block dynamics due to contact phenomena. Int. J. Veh. Mech. Mob., 49: 855-869. DOI: 10.1080/00423114.2010.497841

Grosch, K.A., 1963. The relation between the friction and visco-elastic properties of rubber. Proc. R. Soc. London Series A, 274: 21-39. DOI: 10.1098/rspa.1963.0112 
Sridharan, K. and R. Sivaramakrishnan / American Journal of Engineering and Applied Sciences 5 (2) (2012) 119-127

Majumdar, S., 2011. Tyre tread pattern. Rubber Chem. Rev., 84: 25-35.

Moldenhauer, P. and M. Kroger, 2010. Simulation and experimental investigations of the dynamic interaction between tyre tread block and road. Elastomer Friction Lecture Notes Applied Comput. Mech.,, 51: 165-200. DOI: $10.1007 / 978-3-642-10657-66$

Moore, D.F., 1972. The friction and lubrication of elastomers. 1st Edn., Pergamon Press, New York, pp: 288.
Nakajima, Y., 2007. Numerical simulation of tire traction on various road conditions. Rubber Chem. Technol., 80: 412-435. DOI: 10.5254/1.3548173

Natchimuthu, N., 2010. AFM studies on silica dispersion in EPDM rubber. Rubber Chem. Technol., 83: 123132. DOI: $10.5254 / 1.3548270$

Schallamach, A., 1971. How does rubber slide? Wear, 17: 301-312. DOI: 10.1016/0043-1648(71)90033-0 